\section{ORIGINAL RESEARCH}

M. Mull

R.J. Nijenhuis

W.H. Backes

T. Krings

J.T. Wilmink

A. Thron

\title{
Value and Limitations of Contrast-Enhanced MR Angiography in Spinal Arteriovenous Malformations and Dural Arteriovenous Fistulas
}

\author{
BACKGROUND AND PURPOSE: The purpose of this work was to study the validity of MR angiography \\ (MRA) for identification of spinal arteriovenous (AV) abnormalities.
}

\begin{abstract}
MATERIALS AND METHODS: Thirty-four consecutive patients with suspicion of spinal vascular abnormalities underwent digital subtraction angiography (DSA) after MRA. The level and side of the suspected spinal dural arteriovenous fistula (SDAVF) and the feeding arteries in spinal arteriovenous malformations (SAVMs) were determined from the MRA and compared with DSA.
\end{abstract}

\begin{abstract}
RESULTS: DSA revealed SDAVF in 20 abnormalities of which 19 were spinal and 1 was tentorial with spinal drainage, as well as SAVM in 11 patients. In 3 patients, MRA and DSA were both normal. For detection of spinal arteriovenous abnormalities, neither false-positive nor false-negative MRA results were obtained. The MRA-derived level of the feeding artery in SDAVF agreed with DSA in 14 of 19 cases. In 5 cases, a mismatch of 1 vertebral level (not side) was noted for the feeding artery. For the tentorial AVF, only the spinal drainage was depicted; the feeding artery was outside the MRA field of view. In intradural SAVM, the main feeding artery was identified by MRA in 10 of 11 patients. MRA could differentiate between glomerular and fistulous SAVM in 4 of 6 cases and between sacral SDAVF and filum terminale SAVM in 2 of 5 cases.
\end{abstract}

CoNCLUSIONS: MRA reliably detects or excludes various types of spinal AV abnormalities and localizes the (predominant) arterial feeder of most spinal AV shunts. Although classification of the subtype of SAVMs remains difficult, with MRA it greatly helps to focus subsequent DSA.

A rteriovenous (AV) shunts of the spinal cord and its meninges (ie, spinal AV malformations [SAVMs] and spinal dural AV fistulas [SDAVFs]) are rare and still underdiagnosed entities. If not treated properly, AV shunts can lead to considerable morbidity with progressive spinal cord symptoms and myelopathy because of venous congestion. Usually MR imaging is sufficient to raise the suspicion of a spinal vascular malformation. However, spinal digital subtraction angiography (DSA) composing selective arterial catheterization is still regarded as the only technique sufficiently reliable for the pretreatment detection and characterization of such vascular malformations. ${ }^{1-3}$ Despite the superior image quality of DSA, there are a number of drawbacks in using this imaging technique. Even in experienced hands, selective catheterization of arteries feeding the spinal cord carries the risk of neurologic complications. ${ }^{4}$ The most important drawback is related to the fact that the location(s) of the artery(ies) supplying the AV shunt is (are) not known before angiography, and there are no MR-based predictors (such as location and extent of the cord edema) of the level of the shunt. ${ }^{5}$ Therefore, to localize the possible feeder(s), selective catheterization of all of the arteries potentially supplying the spinal cord and its meninges may be required. These include vertebral, costocervical, and thyrocer-

\section{Received September 21, 2006; accepted after revision November 20.}

From the Department of Neuroradiology (M.M., T.K., A.T.), University Hospital, RWTH Aachen University, Aachen, Germany; Departments of Radiology (R.J.N., W.H.B., J.T.W.) and Surgery (R.J.N.), Maastricht University Hospital, Maastricht, the Netherlands.

Paper previously presented at: Annual Meeting of the American Society of Neuroradiology, May 21-27, 2005; Toronto, Ontario, Canada.

Address correspondence to Walter $\mathrm{H}$. Backes, Department of Radiology, Maastricht University Hospital, P. Debyelaan 25, 6229 HX Maastricht, the Netherlands; e-mail: wbac@rdia.azm.nl

DOl 10.3174/ajnr.A0612 vical trunks, all intercostal lumbar and iliolumbar arteries. ${ }^{5}$ This can lead to often lengthy and sometimes multiple catheterization procedures with high radiation exposures in which large volumes of potentially nephrotoxic contrast agents are administered. ${ }^{6}$

For these reasons, spinal cord $\mathrm{MRA}^{7}$ is evolving as a potentially attractive tool for the detection and evaluation of spinal cord vascular pathology before the DSA examination. In the last decade, several MRA techniques have been developed. First, blood flow-dependent techniques, such as phase conventional angiography $(\mathrm{PCA})^{8}$ and $3 \mathrm{D}$ contrast-enhanced time-of-flight imaging, ${ }^{9}$ were used. These techniques could confirm the presence of abnormally dilated spinal cord arteries or abnormal intraspinal veins, respectively. However, they failed to localize the site of shunt surgery in SDAVF because of the limited temporal resolution and long acquisition time. Binkert et $\mathrm{al}^{10}$ were the first who realized a fast (24-second) contrast-enhanced MRA technique that was able to depict the feeding artery to the SDAVF or SAVM. Although the necessity for a fast MRA technique was recognized, ${ }^{6,10,11}$ it was not yet possible to depict normal intradural arteries, which is mandatory to detect and preserve the specific blood supply to the spinal cord in case of treatment. In addition, detection of feeders of AV shunts in a single MRA session was still limited by the small size of the craniocaudal field of view (FOV). ${ }^{11}$ Because feeding arteries to spinal vascular shunts may arise from all of the arteries potentially supplying the spinal cord, a technique with the largest possible FOV seems therefore necessary to screen for any kind of vascular malformation. Other than detection, it is also important to characterize the subtypes of spinal AV abnormalities, because they are subject to different therapeutic approaches. ${ }^{5}$

In the present study, patients with a suspected spinal AV 
abnormality were examined with contrast-enhanced MRA featuring a large $(50-\mathrm{cm})$ craniocaudal FOV and centric $k$ space sampling, which enabled the visualization of both normal and pathologic arteries and veins. The MRA images were compared with DSA with regard to the following features: detection of the presence of a spinal AV shunt, localization of its arterial feeder(s), and characterization of the type of AV malformation.

\section{Materials and Methods}

\section{Patients}

Between December 2003 and April 2006, 34 consecutive patients (31 men and 3 women) with suspected spinal AV shunts were investigated in 2 centers (Aachen University Hospital, 28 patients; Maastricht University Hospital, 6 patients). The mean age was 59 years (range, 29-78 years). Referral to MRA was based on clinical data and previous MR imaging findings, raising the suspicion of a SAVM (ie, cord edema and/or perimedullary enlarged vessels). In addition to conventional structural MR imaging of the entire spinal cord, all of the patients underwent a single MRA examination as described below during their routine diagnostic work-up before DSA. The interval between MRA and first DSA examination ranged from 1 to 4 days.

\section{Imaging Modalities}

MRA. Imaging was performed on a clinical 1.5T MR imaging system equipped with a phased array spine coil. Patients were imaged in the supine position. The MR imaging protocol was composed of 3 different pulse sequences. ${ }^{12}$ Typically, the MR imaging examination took 30 minutes to perform. First, T2-weighted survey images were acquired to depict the course of the spinal cord as an anatomic reference. Survey scans were acquired in axial and sagittal orientations. Pulse sequence parameters were TR at $2686 \mathrm{~ms}, \mathrm{TE}$ at $120 \mathrm{~ms}$, flip angle (FA) at $90^{\circ}$, FOV at $500 \mathrm{~mm}$, matrix at $320 \times 480$ (phase encoding $\times$ frequency encoding), and 12 4-mm-thick contiguous sections.

Second, MR fluoroscopy was used to determine the optimal scan delay between contrast injection and the start of the MRA acquisition. For this purpose a 2-mL test bolus of gadolinium-based contrast agent (gadopentetate dimeglumine; vial concentration, $0.5 \mathrm{~mol} / \mathrm{L}$ ) was administered. The test bolus was administered at the same injection rate $(3 \mathrm{~mL} / \mathrm{s})$ as the final bolus using a power injector followed by a 25-mL saline flush. The MR fluoroscopy sequence consisted of a single 80 -mm-thick section, which was sagittally positioned through the aorta. This section was acquired once each second for 2 minutes. The pulse sequence parameters were TR at $5.2 \mathrm{~ms}$, TE at $1.4 \mathrm{~ms}$, FA at $35^{\circ}$, FOV at $450 \mathrm{~mm}$, and matrix at $205 \times 256$. Viewing the series of acquired bolus timing images, the scan delay was defined as the time between start of test bolus injection and maximal enhancement of the entire aorta down to the bifurcation. The obtained scan delay was used to synchronize the sampling of the center of $k$-space with the peak contrast agent concentration in the subsequent MRA acquisition.

To facilitate subtraction MRA, 4 consequent precontrast phases were acquired composed of exactly the same pulse sequence parameters as the contrast-enhanced MRA sequence. These precontrast scans were (automatically) averaged to reduce noise propagation, which is inherent to the subtraction of precontrast from contrast-enhanced images, to enhance small vessel conspicuity. Finally, a dynamic 2-phase 3D fast-spoiled gradient echo pulse sequence with $45 \mathrm{~mL}$ of contrast agent was used for MRA. The average precontrast images were subtracted from the corresponding images of the first contrastenhanced phase. The 2 phases served to distinguish relatively early contrast enhancement, which mainly involves (normal and/or pathologic) arteries and pathologic (ie, arterialized) veins, from later enhancement where arteries and arterialized veins but also normal veins are visualized together. Each contrast-enhanced MRA phase took $36-40$ seconds, depending on the number of sections required to cover the entire axial cross-section of all of the vertebrae included. Imaging was performed without breath-hold. FOV was $500 \mathrm{~mm}$ in craniocaudal (frequency-encoding) direction, matrix dimensions were $464 \times 512$ (phase-encoding $\times$ frequency-encoding), and acquired pixel size was $0.9 \times 0.9 \mathrm{~mm}$. Acquired sagittal sections were $1.2-\mathrm{mm}$ thick and interpolated (using 0 -filling in $k$-space) during reconstruction to $0.6-\mathrm{mm}$-thick sections. The number of sections was individually adjusted (range, $75-85 ; 45-51 \mathrm{~mm}$ ) to include the entire vertebral column usually from T3 down to S5 in the (craniocaudal) FOV. Contrast parameters of the fast-spoiled gradient echo pulse sequence were TR of $5.9 \mathrm{~ms}$, TE of $1.9 \mathrm{~ms}$ (partial echo, 62.5\%), and FA of $30^{\circ}$. To emphasize the arterial phase of the bolus enhancement relative to the venous enhancement, $k$-space was sampled using elliptical centric ordering in which the first 4 seconds were randomly filled. This centric ordering of $k$-space sampling permitted a higher (effective) temporal resolution than that of the entire acquisition time of 1 dynamic phase (36-40 seconds) and allowed separation into arterial and mixed arterial-venous enhancement.

Catheter Angiography. Selective IA spinal DSA was performed via a femoral approach in a dedicated biplanar neurangiographic suite. All of the DSA examinations were performed under local anesthesia. Standardized angiography included selective manual injections of $4-5 \mathrm{~mL}$ of $300 \mathrm{mg} / \mathrm{mL}$ of iodinated nonionic contrast medium into lumbar and intercostals arteries. If necessary, additional injections into both vertebral arteries, the costocervical arteries, the thyrocervical trunks, and the iliolumbar arteries was added. Imaging was in the anteroposterior direction with 2 frames per second. X-ray parameters were $80 \mathrm{kV}, 400 \mathrm{~mA}$, matrix of $1024 \times 1024$, and entrance format of $11 \times 13 \mathrm{~cm}$ yielding a spatial resolution of 2.2 line pairs per millimeter. Oblique and lateral views were added to elucidate the morphology of the fistula zone in SDAVF and the composition of the nidus in SAVM. Film sequences of at least 5-20 seconds were taken, depending on the underlying vascular pathology, to evaluate the venous drainage.

In all of the cases of SAVM, the DSA examination included identification of all of the arteries supplying the thoracolumbar cord. If the preceding MRA examination suggested the level of a SDAVF, spinal conventional angiography included at least injection of the segmental arteries, on both sides, 1 level above and below the suggested level. Selective additional injections of the iliolumbar arteries were necessary in patients with suspected spinal sacral AV shunt. In some patients with extensive thoracolumbar venous congestion, complete spinal angiography was necessary with imaging of the cervical and upper thoracic spine via bilateral vertebral, subclavian, costocervical, thyrocervical, and carotid arteries. More than 1 diagnostic DSA examination after MRA was only required in those 3 patients who had negative MRA results.

Image Analysis. The MRA images were postprocessed using maximum intensity projections (MIPs) and multiplanar reformation (MPR). One investigator determined the level and side of the arterial feeder of the AV abnormality ${ }^{12}$ and subsequently provided digital images in which the meningoradicular artery supplying the SDAVF or the arterial feeder(s) of the SAVM were displayed optimally for the 
Table 1: Dural arteriovenous fistula: Findings at MRA and DSA

\begin{tabular}{|c|c|c|c|c|c|c|}
\hline Case No. & Sex/Age (y) & DSA Level & Side & MRA Level & Side & $\begin{array}{l}\text { Level and Side } \\
\text { Correct at MRA }\end{array}$ \\
\hline 1 & $M / 69$ & $\mathrm{~L} 1$ & $\mathrm{~L}$ & $\mathrm{~L} 1$ & $\mathrm{~L}$ & Yes \\
\hline 2 & $M / 63$ & T6 & $\mathrm{L}$ & T6 & $\mathrm{L}$ & Yes \\
\hline 3 & $F / 75$ & T5 & $\mathrm{R}$ & T5 & $\mathrm{R}$ & Yes \\
\hline 4 & $M / 38$ & $\mathrm{~T} 7$ & $\mathrm{~L}$ & $\mathrm{~T} 7$ & $\mathrm{~L}$ & Yes \\
\hline 5 & $\mathrm{M} / 64$ & $\mathrm{~S} 1 / 2$ & $\mathrm{R}$ & $\mathrm{S} 1 / 2$ & $\mathrm{R}$ & Yes \\
\hline 6 & $M / 61$ & T6 & $\mathrm{R}$ & $\mathrm{T7}$ & $\mathrm{R}$ & No \\
\hline 7 & $\mathrm{M} / 71$ & $\mathrm{~T} 6 / \mathrm{T}^{*}$ & $\mathrm{~L}$ & $\mathrm{~T} 6 / \mathrm{T7}^{*}$ & $\mathrm{~L}$ & Yes \\
\hline 8 & $\mathrm{M} / 72$ & T12 & $\mathrm{R}$ & T12 & $\mathrm{R}$ & Yes \\
\hline 9 & $M / 66$ & L3 & $\mathrm{L}$ & L3 & $\mathrm{L}$ & Yes \\
\hline 10 & $\mathrm{M} / 72$ & L1 & $\mathrm{R}$ & L1 & $\mathrm{R}$ & Yes \\
\hline 11 & $\mathrm{M} / 70$ & T5 & $\mathrm{R}$ & T5 & $\mathrm{R}$ & Yes \\
\hline 12 & $\mathrm{M} / 66$ & T6 & $\mathrm{R}$ & T6 & $\mathrm{R}$ & Yes \\
\hline 13 & $\mathrm{M} / 61$ & T8 & $\mathrm{R}$ & T8 & $\mathrm{R}$ & Yes \\
\hline 14 & $M / 56$ & T5 & $\mathrm{R}$ & T6 & $\mathrm{R}$ & No \\
\hline 15 & $\mathrm{M} / 55$ & $\mathrm{~T} 11$ & $\mathrm{R}$ & $\mathrm{T} 10$ & $\mathrm{R}$ & No \\
\hline 16 & $M / 66$ & $\mathrm{~T} 11$ & $\mathrm{~L}$ & $\mathrm{~T} 10$ & $\mathrm{~L}$ & No \\
\hline 17 & $\mathrm{M} / 50$ & $\mathrm{~T} 10$ & $\mathrm{~L}$ & $\mathrm{~T} 11$ & $\mathrm{~L}$ & No \\
\hline 18 & $M / 68$ & $\mathrm{~S} 1 / 2$ & $\mathrm{~L}$ & $\mathrm{~S} 1 / 2$ & $\mathrm{~L}$ & Yes \\
\hline $19 * *$ & $\mathrm{M} / 77$ & L2 & $\mathrm{R}$ & L2 & $\mathrm{R}$ & Yes \\
\hline $20^{* * *}$ & $\mathrm{M} / 63$ & Tentorial & & Outside FOV & & Not imaged \\
\hline
\end{tabular}

Note:-MRA indicates MR angiography; DSA, digital subtraction angiography; $M$, male; $F$, female; $L$, left; $R$, right.

* The fistula was located at T7 left, supplied by the segmental arteries T6 and T7.

** Epidural spinal arteriovenous fistula.

*** Tentorial dural arteriovenous fistula. The fistula was located outside the FOV, but the venous drainage was clearly depicted with MRA.

\begin{tabular}{|c|c|c|c|c|c|c|c|}
\hline \multirow[b]{2}{*}{ Case No. } & \multirow[b]{2}{*}{ Sex/Age (y) } & \multirow[b]{2}{*}{ Location } & \multicolumn{2}{|c|}{ DSA } & \multicolumn{2}{|c|}{ MRA } & \multirow{2}{*}{$\begin{array}{c}\text { AVM Type } \\
\text { Correct a } \\
\text { MRA }\end{array}$} \\
\hline & & & $\begin{array}{c}\text { Dominant } \\
\text { Feeder }\end{array}$ & $\begin{array}{c}\text { Other } \\
\text { Feeders }\end{array}$ & $\begin{array}{c}\text { Dominant } \\
\text { Feeder }\end{array}$ & $\begin{array}{c}\text { Other } \\
\text { Feeders }\end{array}$ & \\
\hline \multicolumn{8}{|c|}{ Glomerular type $(n=3)$} \\
\hline 21 & $M / 39$ & Thoracolumbar & T12 I & T11 I, L1 I & T12 I & & No \\
\hline 22 & $M / 48$ & Thoracolumbar & L1 r & $\mathrm{T} 11 \mathrm{I}, \mathrm{T} 12 \mathrm{r}$ & L1 r & $\mathrm{T} 11 \mathrm{l}, \mathrm{T} 12 \mathrm{r}$ & Yes \\
\hline 23 & $M / 36$ & Thoracolumbar & T9 I & L2 I & T9 I & L2 I & Yes \\
\hline \multicolumn{8}{|c|}{ Fistulous type $(n=3)$} \\
\hline 24 & $F / 43$ & Thoracolumbar & L1 I & T11 r, L1 r, T12 I & L1 I & & Yes \\
\hline 25 & $M / 35$ & Thoracic & T5 r, T6r & & T5 r, T6 r & & No \\
\hline 26 & $M / 29$ & Thoracolumbar & T11 r & & T11 r & & Yes \\
\hline \multicolumn{8}{|c|}{ Filum terminale AVM $(n=5)$} \\
\hline 27 & $M / 56$ & S4/5 & L1 r & Iliaca interna I & L1 r & & Yes \\
\hline 28 & $M / 44$ & L3 & $\mathrm{T} 12 \mathrm{I}$ & & $\mathrm{T} 12$ । & & Yes \\
\hline 29 & $\mathrm{M} / 77$ & L4 & L1 I & & L1। & & No \\
\hline 30 & $\mathrm{M} / 78$ & S2 & L3 I & & L3 I & & No \\
\hline 31 & $M / 61$ & L5/S1 & T11 । & & L4 r & & No \\
\hline
\end{tabular}

Note:-MRA indicates MR angiography; DSA, digital subtraction angiography; $M$, male; $F$, female; AVM, arteriovenous malformation.

comparison study with DSA. The MIP images composed the early and late phases. DSA images were documented on plain films.

In all of the patients, MRA images were analyzed before the DSA examination. The report specified whether the MRA was positive or negative for a spinal AV abnormality. Moreover, the Adamkiewicz artery was localized, ${ }^{13}$ and the spinal AV abnormality was characterized to differentiate between SDAVF (dural or epidural; Table 1) and SAVM (Table 2). Intradural SAVMs were further subcategorized in 1 of 4 groups depending on the morphology, location, and hemodynamics of the AV shunt: 1) fistulous-type SAVM, 2) glomerular-type SAVM, or 3) SAVMs of the filum terminale. SAVMs on the filum terminale are characterized by an abnormally dilated anterior spinal artery and an extremely distant AV shunt located on the filum terminale. If MRA was positive in suspected SDAVF, the side and segmental level of the fistula and, in cases of suspected SAVM, the side and segmental level of the arterial feeders were searched for. When possible, dominant and nondominant feeders were discriminated in SAVM.
Definite localization and characterization of the vascular pathology was based on DSA findings. Two neuroradiologists experienced with spinal vascular abnormalities evaluated the catheter angiograms for the presence and location of the fistula, including the level and side of the SDAVF. For SAVM, the feeding arteries were evaluated with regard to their level and side, their role in supplying the SAVM (dominant versus nondominant), and whether the SAVM was glomerular or purely fistulous. Because pure glomerular or fistulous SAVMs are rare, the dominating feature defined the type of SAVM.

\section{Results}

\section{Detection of AV Abnormalities}

AV shunts were present in 31 of 34 patients, of which 30 were spinal and 1 was tentorial with spinal drainage. MRA detected enlarged perimedullary blood vessels in all 31 patients with spinal abnormalities diagnosed by DSA. No spinal vascular 


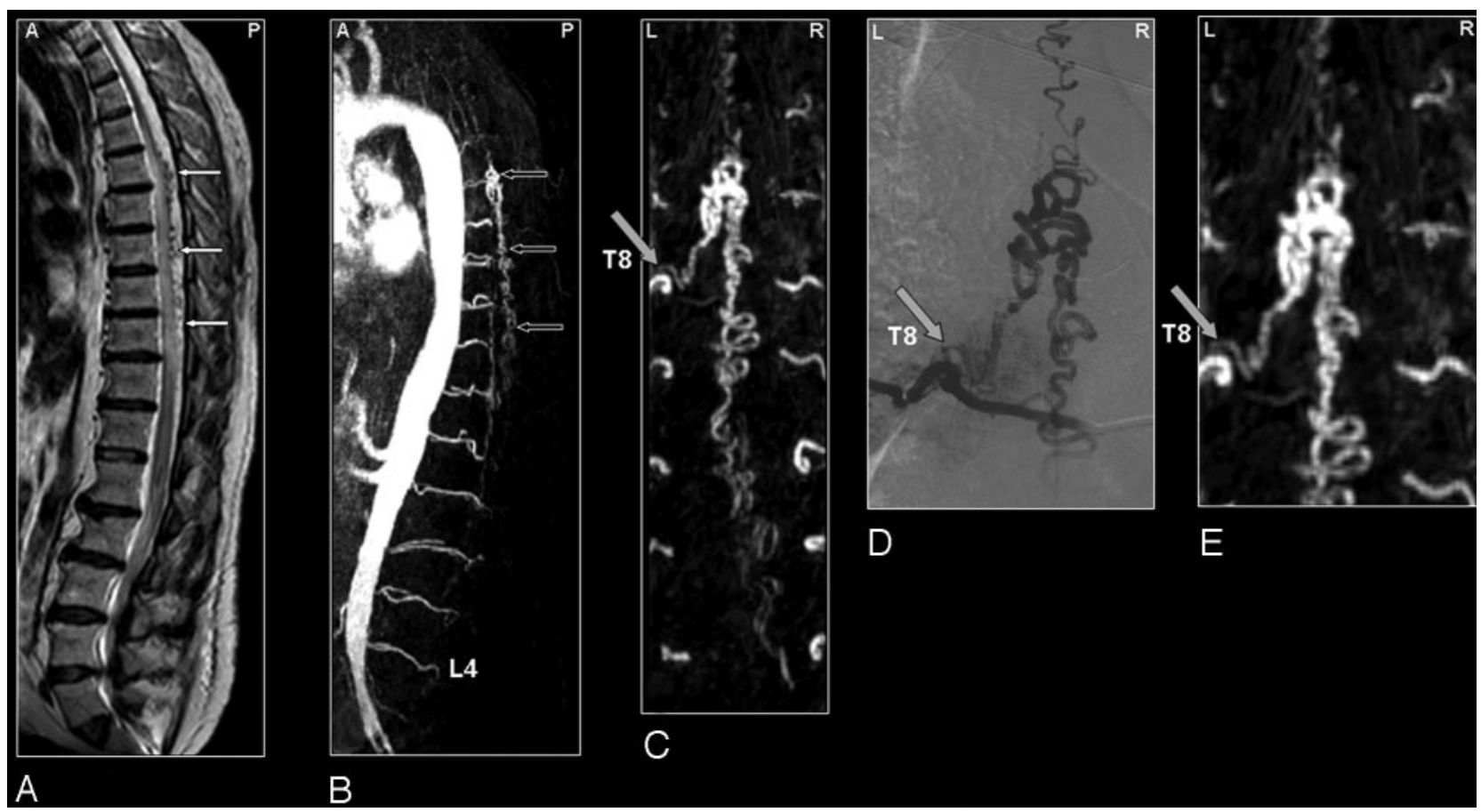

Fig 1. SDAVF in a 61-year-old patient. Comparison of visualization capabilities of MRA and DSA.

A, Sagittal T2-weighted image showing signal intensity voids raising the suspicion of a vascular spinal cord abnormality (small white arrows).

$B$, Sagittal MIPs of the MRA examination showing the overview and localization of the dilated vein (small black arrows).

$C$, In the coronal target MRA MIP the feeding segmental artery of the SDAVF was depicted to derive from the eighth thoracic level (T8) on the left side. Localization of the shunt is at dural level (gray arrow).

DSA $(D)$ provides more spatial resolution and more insight in the dynamic drainage of the dilated vein compared with MRA $(E)$.

abnormalities were found in 3 patients, 2 of whom had a spinal subarachnoid hemorrhage and 1 who had extensive thoracic cord edema suspected to be because of venous congestion. In these 3 patients, complete DSA required up to 4 sessions because of the limitations on the maximum dosage of the contrast medium. No false-positive MRA results were observed in these 3 patients. In 1 patient, a tentorial DAVF was finally encountered. In this subject, the location of the fistula could not be identified by MRA. The extent of the venous drainage visualized by MRA suggested that the fistula was located cranially to the FOV of MRA. The subsequent DSA examination demonstrated a tentorial DAVF with extensive spinal perimedullary venous drainage.

One patient was classified with an epidural AVF. The level of the arterial lumbar feeder in this patient could be defined after MRA pointed to the correct level resulting in a successful DSA examination.

\section{Localization of SDAVF}

In the 19 patients with SDAVF with retrograde perimedullary venous spinal drainage, MRA correctly predicted the level of the fistula within 1 vertebral level in all of the subjects (Table 1 and Figs 1 and 2). Even deeply located sacral fistulas were correctly identified by MRA before DSA (Fig 3). In 5 subjects, a mismatch of 1 vertebral level (not side) was noted (Fig 4). Possible reasons for these mismatches were the poorly visualized proximal part of the draining vein (patients 6,14, and 16) or variants of the segmental arterial supply and the bony structures (patients 15 and 17).

\section{Arterial Feeders in SAVM}

Eleven patients were identified with an SAVM, which were localized in the thoracolumbar region in 6 subjects (Fig 5) and on the filum terminale in 5 subjects (Fig 6). Fistulous-type SAVMs were found in 3 patients. Five patients had an AVM of the filum terminale. Three patients had a glomerular-type SAVM with a distinct nidus of abnormal vessels between the arterial and venous side. This nidus could be seen clearly in the parenchymal MR image. MRA and DSA findings are listed in Table 2.

The main arterial feeder could be identified in 10 of 11 cases of SAVM with MRA. There were 5 patients with additional feeders demonstrated by DSA. MRA could only depict additional feeders in 2 patients (patients 22 and 23).

In the thoracolumbar region, differentiation between glomerular and fistulous SAVM was successful with MRA in 4 of 6 patients. For the filum terminale SAVM, the dilated main arterial feeder was found in 5 of the 5 patients. However, classification of this rare type of abnormality by MRA was only possible in 2 patients. In the remaining 3 patients, DSA was necessary to correctly classify this abnormality.

\section{Discussion}

\section{Current Findings}

This study has shown to what extent contrast-enhanced MRA may serve to detect, localize, and characterize various types of spinal AV shunts in patients with suspected AV malformations, with selective spinal angiography as the reference study. 


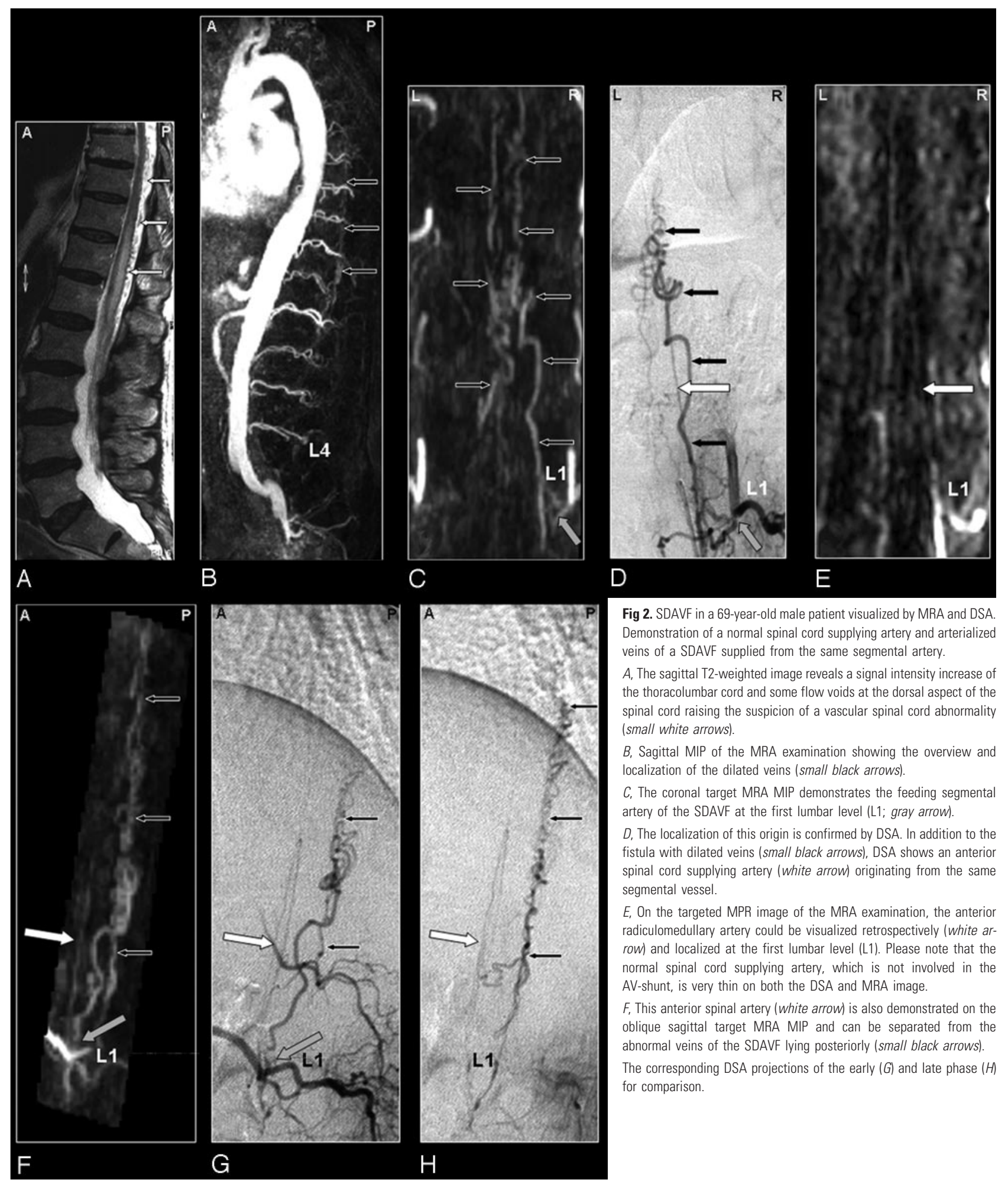

The most important finding of this study was the observation that no false-positive and no false-negative MRA results were obtained regarding AV shunts of the spinal cord in a relatively large series of patients who harbor a rare disease. Consequently, because detection or exclusion of spinal AV abnormalities by MRA was found to be very accurate for both SDAVF and SAVM, it may in the future be possible to avoid burdensome diagnostic conventional angiography proce- dures. Furthermore, MRA may substitute for DSA for follow-up diagnostic procedures.

One may argue that one patient, who had a (cranial) tentorial dural AV fistula, was not correctly diagnosed by MRA. However, MRA visualized the associated pathologically arterialized veins and guided the subsequent DSA examination to the cranium. In this scan protocol, we did not include the cervical spine for 2 reasons: there is a technical restriction in the number of coil ele- 


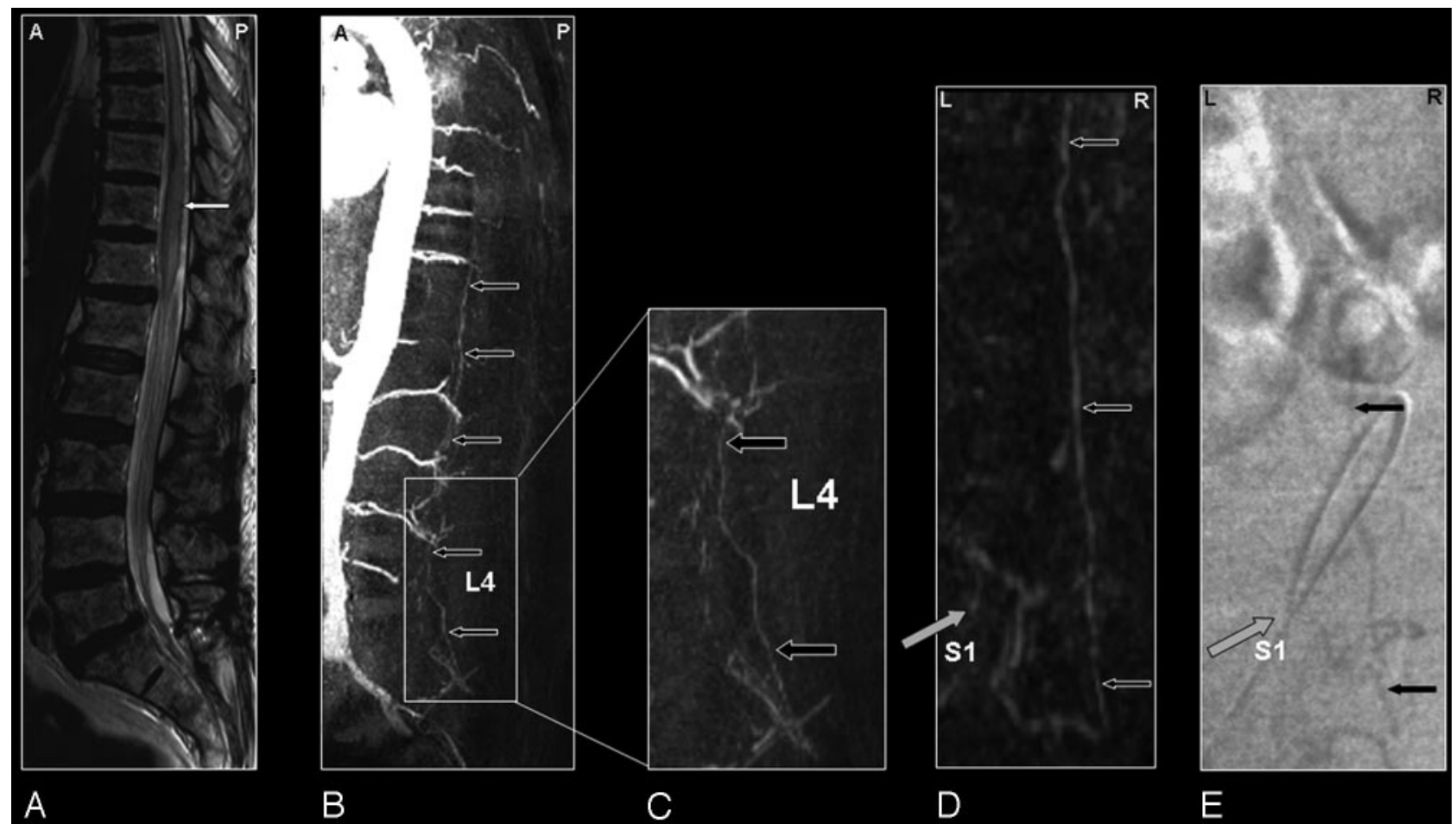

Fig 3. Sacral SDAVF in a 64-year-old male patient visualized by MRA and DSA. Advantage of MRA in demonstrating vascular abnormality in a large FOV.

A, Sagittal T2-weighted image showing extensive spinal cord edema and only some enlarged vessels raising the suspicion of a vascular spinal cord abnormality (small white arrows). $B$, Sagittal MIP of the MRA examination showing the overview and localization of an early filled and dilated vein (small black arrows).

$C$, In the enlarged view, the dilated vein of the filum terminale is more clearly depicted (black arrows).

The coronal target MRA MIP shows the arterialized filum vein (D, small black arrows) in the same projection in which DSA ( $E)$ could confirm the localization of the very small shunt at the first sacral level (gray arrow).

ments that can be used at once, and SDAVF of the cervical spine is exceedingly rare. ${ }^{3}$ Therefore, we opted to use the elements that covered the entire thoracic, lumbar, and sacral region.

\section{Diagnostic Spinal MRA}

The possibility of MRA identifying spinal cord vascular pathology was recognized previously in a number of studies. Mascalchi et $\mathrm{al}^{8}$ found PCA suitable for the detection of SDAVFs and the arterial feeders of SAVMs in a high percentage ( $\leq 12$ of 15 ) of patients. Detection of draining veins, localization of the fistula, and localization of normal arteries were, however, limited or in some cases not possible because of their low blood flow or small dimensions. Bowen et al, ${ }^{9}$ on the other hand, by using contrast-enhanced 3D steady-state MRA, were able to visualize the dilated spinal cord veins associated with SDAVFs. Although these pathologic veins pointed toward the site of the AV shunt, the shunt itself was difficult to depict, and normal intradural arteries were not observed. The main reason for this appears to be the fact that the contrast material was distributed over the relatively large veins and adjacent (background) tissue, which obscured the visualization of the small arteries and the shunt surgery zone. Binkert et $\mathrm{al}^{10}$ avoided contaminating venous and background enhancement by applying a fast (24-second) contrast-enhanced MRA technique and showed in a limited number of patients that the localization of the feeder of SDAVFs (2 of 3 patients) and the classification of SAVMs ( 6 of 6 patients) were feasible.

Recently, the studies of Farb et $\mathrm{al}^{11}(n=9)$ and Luetmer et $\mathrm{al}^{6}(n=31)$ demonstrated that SDAVFs can be accurately localized using an MRA technique in which the AV contrast was emphasized with a bolus injection of contrast agent similar to our technique. Unfortunately, the craniocaudal FOV was limited ( $28 \mathrm{~cm}$ and $36 \mathrm{~cm}$, respectively), thereby missing the level of the fistula in 5 patients in the study by Farb et $\mathrm{al}^{11}$ and in 6 patients in the study by Luetmer et al. ${ }^{6}$ In neither study described above was it possible to demonstrate normal intradural arteries because of their small calibers of $1 \mathrm{~mm}$ or less and because of the course, which is similar to perimedullary and radicular veins.

In the present study, we have improved on these previous techniques by consistently visualizing the Adamkiewicz artery and its continuation in the anterior spinal artery by the temporal separation of early phases (arteries and arterialized veins) from relatively late phases of enhancement (arteries and veins together). Moreover, using our technique, it was demonstrated that it is possible to visualize even small abnormal blood vessels of the lumbosacral spine in patients with SDAVF and SAVM of the filum terminale.

The contrast-enhanced MRA technique used in this study is similar to the one used previously for the preoperative detection of the Adamkiewicz artery in patients about to undergo thoracoabdominal aortic aneurysm surgery. ${ }^{14}$ The relevance of detecting the Adamkiewicz artery in spinal AV shunts is illustrated by the case demonstrated in Fig 2, in which the radiculomeningeal artery supplying the SDAVF also gave rise to the Adamkiewicz artery. For this case, cautious treatment is required to avoid interruption of the blood supply to the Adamkiewicz artery, because there is increased risk for developing paraplegia. 


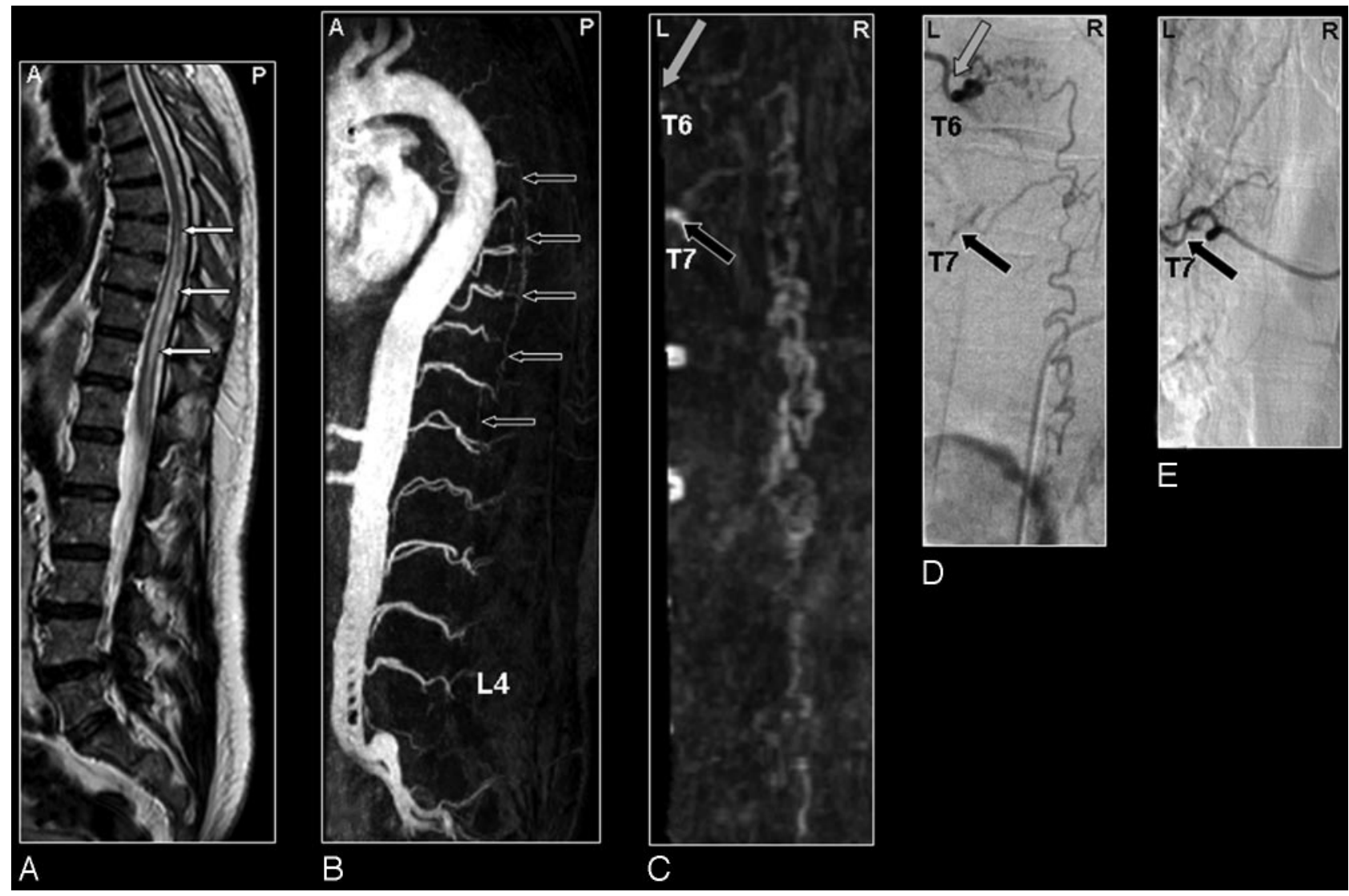

Fig 4. SDAVF in a 61-year-old male patient visualized by MRA and DSA. Misinterpretation of the segmental level of origin on MRA.

A, Sagittal T2-weighted image showing spinal cord edema (small white arrows).

$B$, Sagittal MIP of the MRA examination showing the overview and localization of the dilated vein (small black arrows).

$C$, In the coronal target MRA MIP the feeding segmental artery of the SDAVF was falsely localized by just 1 level at the seventh thoracic level (T7; black arrow).

$D$, DSA shows that the feeding segmental artery (gray arrow) originates from the sixth thoracic level (T6).

E, Selective injection of segmental artery T7 (black arrow) shows no supply to the SDAVF. Retrospectively, the correct level (gray arrow) could be identified on the target MRA MIP (C).

The combination of a relatively large craniocaudal FOV (50 $\mathrm{cm})$, a high gadolinium dose $(0.3 \mathrm{mmol} / \mathrm{kg})$ administered as a bolus, subtraction with noise-reduced precontrast images, and the synchronization of central $k$-space sampling to the contrast bolus arrival provides a more complete diagnostic examination in which normal and abnormal arteries and veins can be distinguished, the AV shunt zone can be visualized, the predominant arterial feeders can be localized, and the venous drainage pattern can be displayed in a single MRA examination.

\section{Limitations}

Despite these improvements, there are limitations. Detection of multiple arterial feeders in cases with extensive fistulous SAVM remains limited to the largest feeders. Small additional feeders remain unnoticed with MRA. Although MRA was able to detect AV abnormalities of the sacral spine, differentiation between a sacral SDAVF or filum terminale SAVM was not possible in all of the cases. Likewise, subclassification of SAVMs in terms of glomerular and fistulous was not successful in all of the cases and still requires conventional angiography.

It should be stressed that the reliability of contrast-enhanced MRA for the purpose described in this study is strongly dependent on the quality of postprocessing (ie, MPR), which is elaborate and time-consuming ( $\leq 1$ hour) and requires considerable skill and dedication. A possible limitation of the cur- rent study is the fact that only a single investigator created the postprocessed MRA images for comparison with DSA. A study involving the use of multiple independent investigators would allow for the assessment of the interobserver variability of the specialized postprocessing steps.

Only 3 patients, who showed no abnormality after performing MRA and conventional angiography, were included. A larger true negative population would increase the clinical value of this comparative study. However, because of the applied selection criteria, which were based on clinical data and previous MR imaging findings, the number of patients who showed no abnormality was quite small.

\section{Future Improvements}

A future possibility to characterize a SAVM more accurately would be the use of a second MRA examination in addition to the first MRA with the large FOV. In the second MRA examination, a smaller FOV could focus on the vascular pathology detected in the first MRA examination and could reveal more details of the fistulous zone and/or arterial feeders.

Future developments that may allow us to improve the MRA examination are the use of high T1-relaxivity (eg, blood pool) contrast agents. This could enable the more detailed depiction of the shunt surgery zone rather than merely localizing the arterial feeder. Whether the use of a blood pool agent 


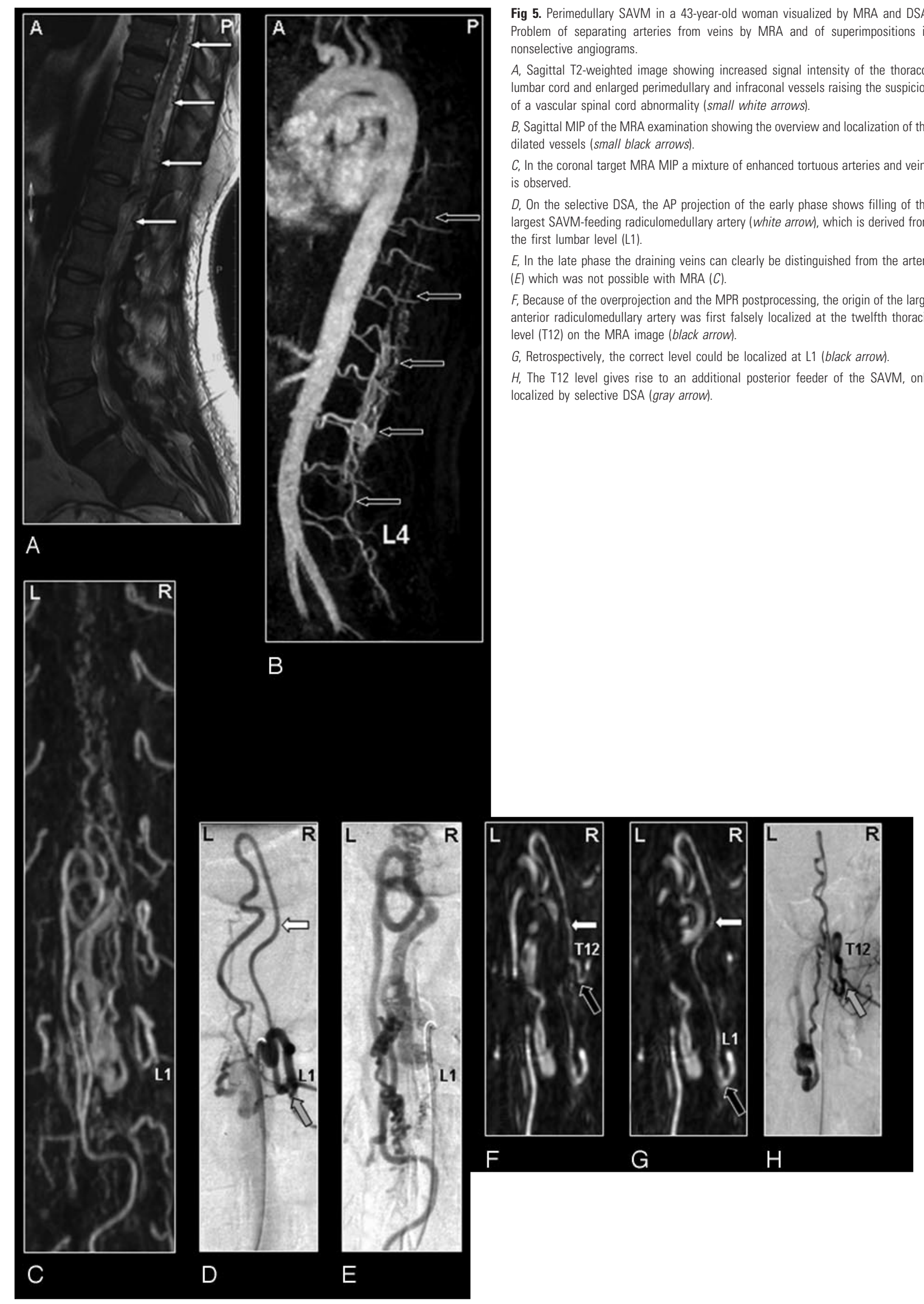



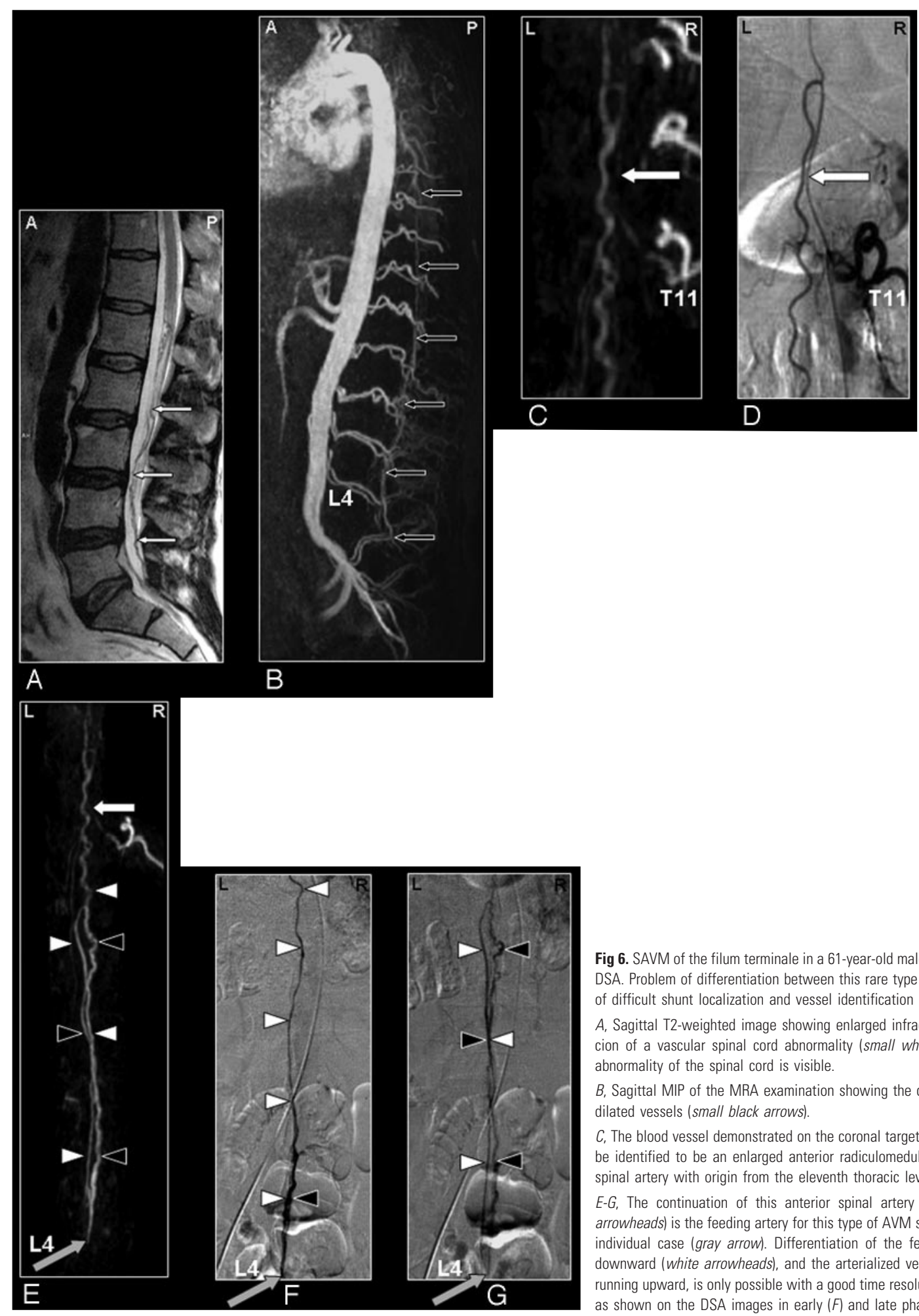

Fig 6. SAVM of the filum terminale in a 61-year-old male patient visualized by MRA and DSA. Problem of differentiation between this rare type of SAVM and SDAVFs because of difficult shunt localization and vessel identification in the present MRA.

A, Sagittal T2-weighted image showing enlarged infraconal vessels raising the suspicion of a vascular spinal cord abnormality (small white arrows); no signal intensity abnormality of the spinal cord is visible.

$B$, Sagittal MIP of the MRA examination showing the overview and localization of the dilated vessels (small black arrows).

$C$, The blood vessel demonstrated on the coronal target MRA MIP could retrospectively be identified to be an enlarged anterior radiculomedullary (white arrow) and anterior spinal artery with origin from the eleventh thoracic level. DSA for comparison $(D)$.

E-G. The continuation of this anterior spinal artery to the filum terminale (white arrowheads) is the feeding artery for this type of AVM situated at the level of L4 in this individual case (gray arrow). Differentiation of the feeding artery, which is running downward (white arrowheads), and the arterialized vein (black arrowheads), which is running upward, is only possible with a good time resolution of the selective angiogram as shown on the DSA images in early $(F)$ and late phases $(G)$.

itself is sufficient to improve the visualization of the small spinal cord vasculature remains uncertain, because the conventional gadolinium contrast material remains within the intravascular compartment because of the blood-cord barrier.

In theory, imaging could improve when changing to a $3 \mathrm{~T}$ magnet, because it offers better signal intensity-to-noise characteristics that can be used to increase spatial and temporal resolution and to better depict smaller vessels. However, there is in our opinion no obvious benefit in changing from a $1.5 \mathrm{~T}$ to a $3 \mathrm{~T}$ mag- net. The major advantage of the $1.5 \mathrm{~T}$ system is the ability to use a dedicated spine coil, which enables a sufficiently homogeneous FOV up to $500 \mathrm{~mm}$ but is not (yet) available for our 3T MR imaging system. The large FOV helps to localize the variable origin of the feeders of the vascular abnormalities, as well as the Adamkiewicz artery, along the entire thoracolumbar-sacral spinal cord. Achieving such a large FOV is not possible with current 3T systems. Moreover, the stronger field inhomogeneities near the vertebral bodies seem to counteract the increase in signal in- 
tensity-to-noise ratio. Therefore, a major technologic challenge is to be taken to improve the 3T MR imaging system for spinal cord angiography.

\section{Conclusion}

We demonstrated that contrast-enhanced MRA is able to visualize both normal and pathologic arteries and veins of the spinal cord. The arterial feeder of a SDAVF can be accurately pinpointed by MRA, but for SAVM only the predominant arterial feeder can be visualized, and subclassification, which is relevant for the choice of treatment, should still be based on conventional angiography. Spinal cord AV abnormalities can be reliably detected or excluded by MRA (ie, $100 \%$ predictive value), which may focus burdensome conventional angiography procedures or even avoid them in cases of normal MRA.

\section{References}

1. Berenstein A, Lasjaunias P. Spine and spinal cord vascular lesions. In: Surgical Neuroangiography. Vol 5. Berlin: Springer-Verlag; 1992:1-85

2. Thron AK. Vascular anatomy of the spinal cord. Wien, New York: SpringerVerlag; 1988:3-5

3. Brandt T, Caplan LR, Dichgans J, et al. Vascular malformations and interventional neuradiology of the spinal cord. In: Brandt T, et al, eds. Neurological Disorders Course and Treatment. 2nd ed. Boston: Academic Press; 2003:517-28

4. Forbes G, Nichols DA, Jack CR Jr, et al. Complications of spinal cord arteriography: prospective assessment of risk for diagnostic procedures. $R a$ diology 1988;169:479-84

5. Krings T, Mull M, Gilsbach JM, et al. Spinal vascular malformations. Eur Radiol 2005; 15:267-78

6. Luetmer PH, Lane JI, Gilbertson JR, et al. Preangiographic evaluation of spinal dural arteriovenous fistulas with elliptic centric contrast-enhanced MR angiography and effect on radiation dose and volume of iodinated contrast material. AJNR Am J Neuroradiol 2005;26:711-18

7. Pattany PM, Saraf-Lavi E, Bowen BC. MR angiography of the spine and spinal cord. Top Magn Reson Imaging 2003;14:444-60

8. Mascalchi M, Quilici N, Ferrito G, et al. Identification of the feeding arteries of spinal vascular lesions via phase-contrast MR angiography with three-dimensional acquisition and phase display. AJNR Am J Neuroradiol 1997;18:351-58

9. Bowen BC, Fraser K, Kochan JP, et al. Spinal dural arteriovenous fistulas evaluation with MR angiography. AJNR Am J Neuroradiol 1995;16:2029-43

10. Binkert CA, Kollias SS, Valavanis A. Spinal cord vascular disease: characterization with fast three-dimensional contrast-enhanced MR angiography. AJNR Am J Neuroradiol 1999;20:1785-93

11. Farb RI, Kim JK, Willinsky RA, et al. Spinal dural arteriovenous fistula localization with a technique of first-pass gadolinium-enhanced MR angiography: initial experience. Radiology 2002;222:843-50

12. Nijenhuis RJ, Leiner T, Cornips EM, et al. Spinal cord feeding arteries at MR angiography for thoracoscopic spinal surgery: feasibility study and implications for surgical approach. Radiology 2004;233:541-47

13. Nijenhuis RJ, Mull M, Wilmink JT, et al. MR angiography of the great anterior radiculomedullary artery (Adamkiewicz artery) validated by digital subtraction angiography. AJNR Am J Neuroradiol 2006;27:1565-72

14. Nijenhuis RJ, Gerretsen S, Leiner T, et al. Comparison of 0.5-M Gd-DTPA with 1.0-M gadobutrol for magnetic resonance angiography of the supplying arteries of the spinal cord in thoracoabdominal aortic aneurysm patients. J Magn Reson Imaging 2005;22:136-44 